\title{
Are rates of vision loss \\ in patients in English \\ glaucoma clinics \\ slowing down over \\ time? Trends from a decade of data
}

T Boodhna, LJ Saunders and DP Crabb

Boodhna, L Saunders and DP Crabb

This article has been corrected since Advance Online Publication and a corrigendum is also printed in this issue

\begin{abstract}
Purpose To examine changes in rates of visual field (VF) progression in patients attending a sample of glaucoma clinics in England between 1999 and 2012.

Methods An archive of 473252 Humphrey

VFs recorded across the UK was

retrospectively examined. Distribution of rates recorded in the first half of the decade was compared with the second. The relationship between age and severity of MD loss at baseline with rates of loss and frequency of testing was examined. Results VF series from 18926 eyes were analysed. Median rate of MD loss for the period before and after 2003 was -0.11 and $-0.06 \mathrm{~dB} /$ year, respectively, but the proportion of eyes with medium or fast rates of MD loss remained constant. Median rate of MD loss in older ( $>70$ years) eyes was faster than that observed in younger $(<60$ years) eyes $(-0.21$ compared with $-0.01 \mathrm{~dB} /$ year). Median rate of loss did not vary with severity of MD loss at baseline. Frequency of testing, typically carried out annually, did not vary by age, rate of loss or disease severity. Conclusions VFs of eyes treated in the first half of the decade deteriorated more rapidly than those in the second half. Several factors might explain these differences but average effects were small and there was no reduction in the proportion of rapidly progressing eyes over the decade. Older age and, to a lesser extent, worse VF damage at diagnosis are indicators for faster VF loss in clinics, but frequency of VF testing was similar for all patients.

Eye (2015) 29, 1613-1619; doi:10.1038/eye.2015.161; published online 28 August 2015
\end{abstract}

Introduction

Rate of visual field (VF) loss determined from a series of examinations in time can be clinically useful in managing a patient with glaucoma. Rates of VF loss vary enormously among patients and can only be determined by observation of individuals. Still, several studies, both prospective and retrospective, have derived estimates of average rate of VF loss in groups of patients. ${ }^{1-3}$ Indeed, knowledge of the distribution of rates of loss in populations as defined by mean deviation (MD) loss per year, where MD is a commonly used metric for assessing severity of VF loss, is clinically useful information. Recent studies, using data from treated patients in routine care, have yielded estimates for median rate of MD loss that vary considerably from -0.05 to $-0.62 \mathrm{~dB} /$ year. ${ }^{1,4}$

Adding to this literature by considering another large cohort of real-world data would be worthwhile. Furthermore, we are unaware of any studies considering how average rates of VF loss may have changed in the same sample of clinics over a significant period of time and this is the main idea explored in this paper.

Routinely collected clinical data can be used to assess real-world outcomes from implementing evidence-based findings from trials. These assessments can be carried out by taking advantage of large data sets collected from electronic patient records, and there are some good recent examples of this type of approach being used in ophthalmology. ${ }^{5-8}$ Automated perimetry has been routinely used in glaucoma clinics for more than 20 years and VF data recorded electronically in many centres can be used to monitor trends in health service delivery of glaucoma. ${ }^{9,10}$
Optometry and Visual Science, School of Health Sciences, City University London, London, UK

Correspondence: DP Crabb, Optometry and Visual Science, School of Health Sciences, City University London, London EC1V OHD, UK

Tel: +044 (0) 207040 0191; Fax: +44 (0) 2070400191. E-mail: david.crabb.1@ city.ac.uk

Received: 14 May 2015 Accepted in revised form: 27 July 2015

Published online: 28 August 2015 
This approach, using large-scale VF data, is adopted in the methodology of this study.

The first decade of the new millennium saw a shift to new topical treatment for glaucoma and ocular hypertension. For example, Owen and colleagues, using data from nearly a half of one million patients registered at 131 general practices across the UK, identified 2003 to be the year that number of prescriptions for prostaglandins overtook beta blocker-only medication. It remains unclear whether the introduction of these treatments impacted on disease progression in patients with glaucoma. ${ }^{11}$ This question is immune to a research study, no matter the experimental design. However, we aim to gather some insight by considering large-scale VF data recorded over a 13-year period in order to test the hypothesis that rates of VF loss differed in patients diagnosed before and after 2003. A secondary aim is to describe the distribution of rates of VF loss stratified by age and severity of VF loss at baseline, along with a consideration of how these strata of patients may be followed more or less frequently during follow-up.

\section{Materials and methods}

As described elsewhere, $, 9,10,12$ Medisoft VF databases (Medisoft Ltd., Leeds, UK) containing 473252 VFs from 88954 patients were downloaded in 2012 from glaucoma clinics at Moorfields Eye Hospital in London, Cheltenham General Hospital Gloucestershire Eye Unit, Queen Alexandra Hospital in Portsmouth and the Calderdale and Huddersfield NHS Foundation Trust. Data access was granted by the Caldicott guardians at each centre. All patient data were anonymised and transferred to a single secure database. No other clinical data were made available apart from patient's age and the dates of the VF examinations. Subsequent analyses of the data were approved by a research ethics committee of City University London and this study adhered to the Declaration of Helsinki.

Only VFs recorded on the Humphrey Visual Field Analyzer (HFA, Carl Zeiss Meditec, Dublin, CA, USA) using a Goldmann size III stimulus with a 24-2 test pattern acquired with the Swedish Interactive Testing Algorithm (SITA Standard or SITA Fast) were included, reducing the data set to 423194 VFs. Series of data from patients $>40$ years recorded between 01 January 1999 and 31 December 2011 were then extracted. (Only those patients measured consistently with SITA Fast or SITA standard were included.)

Eyes with short follow-up (less than 4 years or less than five examinations) were excluded. The first VF examination in each series were then removed from further analysis to account for perimetric learning effects. ${ }^{13-15}$ Precision of estimating the rate of MD loss (dB/year) using simple linear regression varies enormously by the length of follow-up. ${ }^{16}$ We attempted to control for this by only calculating the rate within a fixed 4 -year period (window) from the baseline test (see Figure 1). Each series had to have at least three examinations within this period. Of course, this does not mean we excluded eyes with longer follow-ups. Yet, this fixed window was important for our comparison of rates across the study period because those diagnosed at the start of the study period would have had much longer follow-ups than those towards the end of the study period.

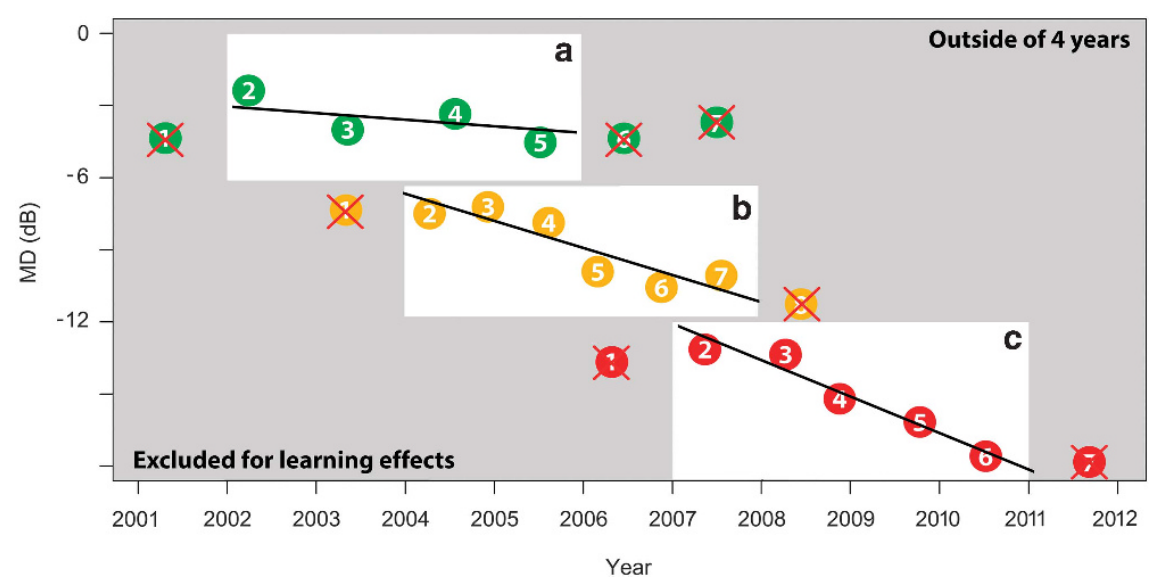

Figure 1 A schematic illustrating the VF series inclusion criteria and method for calculating rates of MD loss (dB/year) for three example eyes detected in 2001 (a), 2003 (b), and 2006 (c). Eyes were excluded if $<5$ VF examinations or $<4$ years of follow-up. The first VF in each series was omitted to account for perimetric learning effects. Rate was calculated from linear regression of the baseline VF and the series of exams that fell within a 4-year period after it (white window). So, for example, for series (a) the sixth and seventh recorded VFs fall outside this window and are not used in the calculation. This ensures that all rates are estimated with equivalent precision allowing for comparisons over time. A minimum of three VFs were required to be in this 4-year window. This rate was then assigned to the date of the baseline exam. 
A total of 18926 eyes met the inclusion and exclusion criteria. These data represent patients in glaucoma clinics who are receiving routine care. Rates of MD loss $(\mathrm{dB} /$ year) were recorded and ranked by date of the baseline test. The data were then simply divided into two parts by chronological order. Thus, distribution of the rates of MD loss from baseline examinations in the first (15.01.99 to $16.09 .03 ; n=9463$ eyes) and second (17.09.03 to $05.09 .08 ; n=9463$ eyes) half of the study period could be compared. An eye, or a patient could only appear in one time period.

Furthermore, eyes with rates of MD loss better than $0 \mathrm{~dB} /$ year were defined as stable; those with rates between 0 and $-0.5 \mathrm{~dB} /$ year were defined as slow rate progressors; those with rates between -0.5 and $-1.5 \mathrm{~dB} /$ year were defined as medium rate progressors, whereas those with rates worse than $-1.5 \mathrm{~dB} /$ year were defined as fast rate progressors. Temporal change in the proportion of patients in these categories was analysed with a conditional density plot; this shows how a categorical variable, in this case stable, slow, medium, and fast progressors, changes over values of a continuous variable (time or estimated date of diagnosis).

Eyes were stratified to determine the relationship between age and severity of MD loss at baseline with rates of VF loss. Eyes were stratified into simple age categories: younger patients ( $<60$ years, $n=6311$ ) and older patients ( $>70$ years, $n=6385$ ). All others were considered to be average age patients $(n=6230)$. Eyes with MDs better than $-6 \mathrm{~dB}$, between $-6 \mathrm{~dB}$ and $-12 \mathrm{~dB}$ or worse than $-12 \mathrm{~dB}$ were categorised as having early $(n=10920)$, moderate $(n=3122)$, or advanced/severe ( $n=2063)$ VF loss, respectively. These values were taken from a widely used criterion for summarising disease stages in glaucoma and are represented within the colour schemes of Figure 1 (mild represented by green, moderate represented by orange, and severe represented by red). ${ }^{17}$

A simple metric for the frequency of examination during the 4-year follow-up period was also calculated. Eyes with three, four, or five examinations in this period were defined as receiving approximately 'annual testing'. All others were considered to be having more frequent VF surveillance. The percentage of eyes that had annual testing was then compared across the disease severity and age strata. This metric was also calculated for eyes with progression rates between 0 and $0.5 \mathrm{~dB} /$ year, 0.5 and $1.5 \mathrm{~dB} /$ year, and worse than $1.5 \mathrm{~dB} /$ year being categorised as slow $(n=5849)$, medium $(n=3774)$, and fast progressors $(n=1123)$, respectively.

All statistical analyses were carried out using the opensource programming language, R. ${ }^{18}$

\section{Results}

In total, VF series from 18926 eyes from 13984 patients were analysed. Median (interquartile range) age, MD at baseline, and number of examinations in the 4-year follow-up were 65.5 (56.7-72.6) years, -2.8 (-6.6 to $-0.7) \mathrm{dB}$, and five (four to five), respectively.

The distribution of rates of MD loss ( $\mathrm{dB} /$ year) in eyes diagnosed in the period 1999-2003 and 2003-2008 are shown in Figure 2. Although the median progression rate of theses distributions are different, indicating that average rates of VF loss slowed in the second period, the lower percentiles (25th and 10th) remained the same suggesting that there was no change in the proportion of patients who are more rapidly progressing. Median (interquartile range) age and MD at baseline in eyes diagnosed in the period 1999-2003 were 65.2 (56.4-72.2) years and $-2.9(-6.7$ to -0.8$) \mathrm{dB}$, respectively. Median (interquartile range) age and $\mathrm{MD}$ at baseline in eyes diagnosed in eyes diagnosed in the period 2003-2008 were $65.8(57.1-73.0)$ years and $-2.6(-6.3$ to -0.7$) \mathrm{dB}$, respectively.

Figure 3 illustrates the change in the proportion of eyes categorised as having stable, slow, medium, or fast VF loss. The percentage figures indicate the change in relative proportions of progressors across the two halves of the
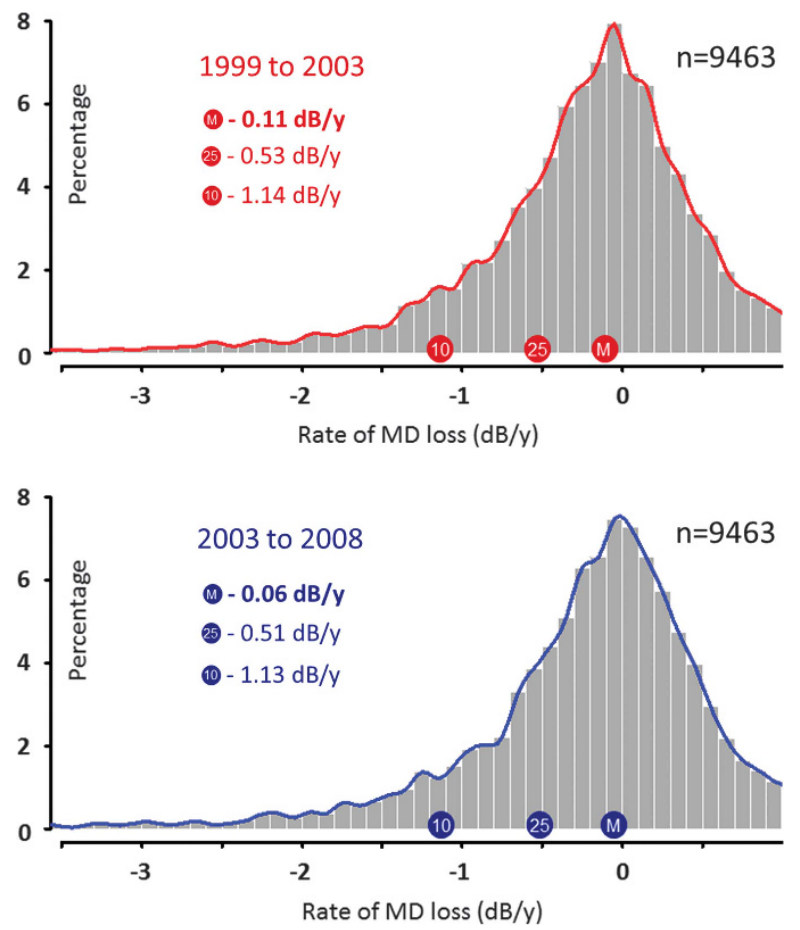

Figure 2 Distribution of MD rate in eyes diagnosed in two periods of the decade. Median, 25th (lower quartile), and 10th percentile are indicated. Curved lines represent a spline fit to the histogram. Note the histogram is censored at $+1 \mathrm{db} /$ year. 


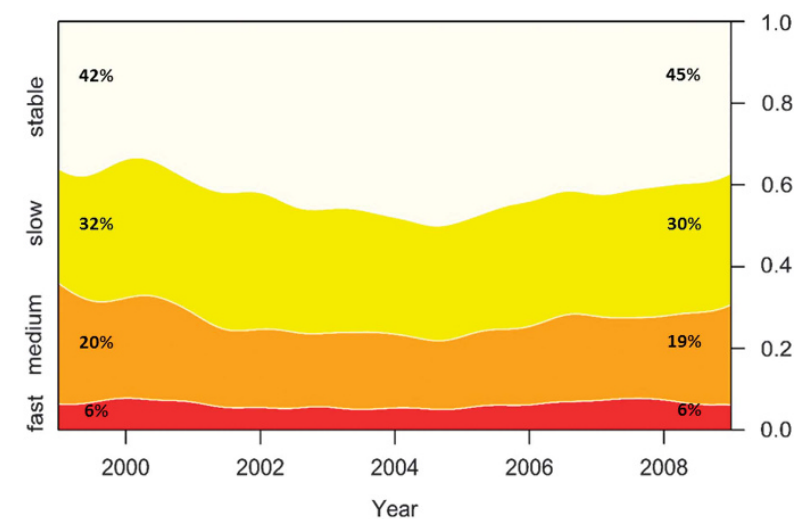

Figure 3 Conditional Density Plot showing the temporal change in the relative proportion of eyes with different rates of VF loss (stable, slow, medium, fast), across the midpoint of the study period. A $3 \%$ increase in the proportion of stable progressors was identified in this study with a $2 \%$ and $1 \%$ reduction identified for the slow and medium progressors, respectively. No change was observed in the fast progressors.
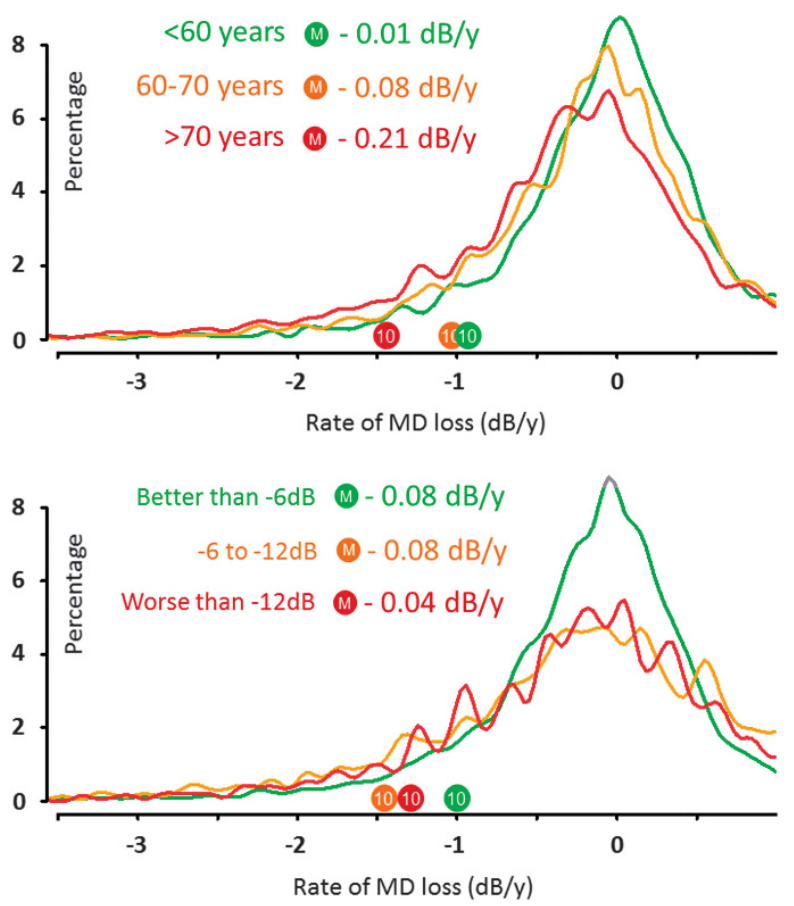

Figure 4 Distribution (spline fit of histogram) of MD rate in eyes grouped by baseline age (top) and baseline severity of VF loss (bottom). Median and 10th percentile values are indicated over the study period.

study period. It is noteworthy that the proportion of eyes defined to be medium or fast progressors remains largely the same over the entire study period.

Figure 4 illustrates the distribution of progression rates by age and disease severity. Older eyes ( $>70$ years) were found to progress considerably faster than younger eyes ( $<60$ years). Median rate of MD loss was similar across

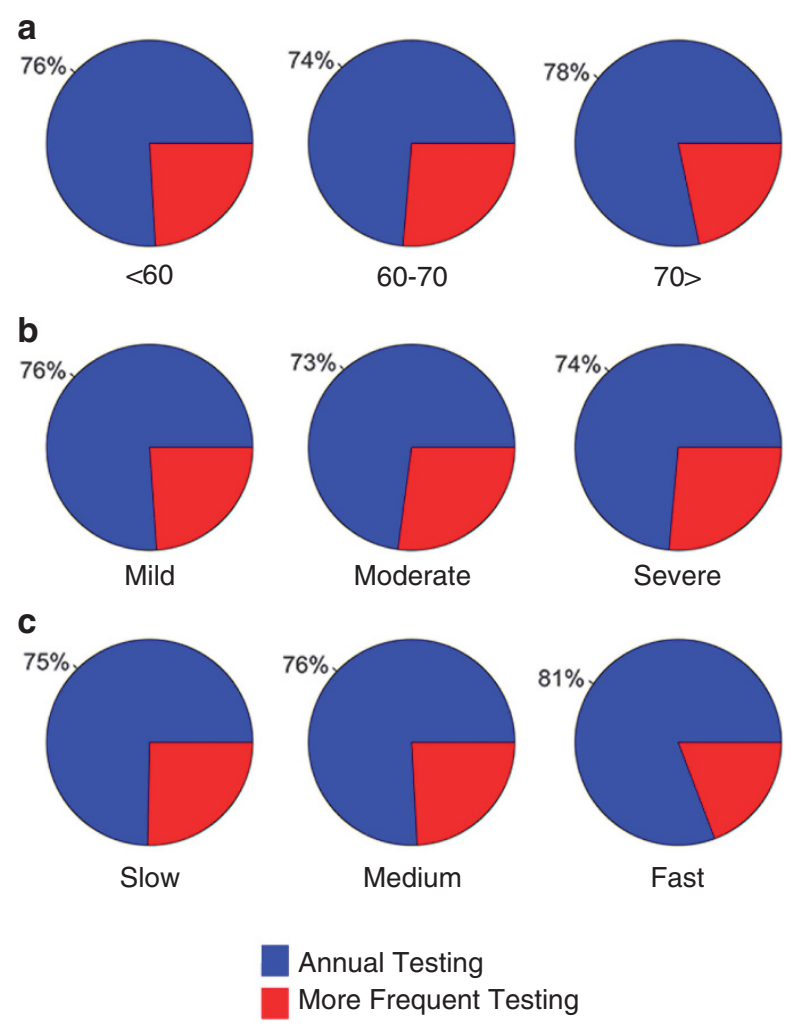

Figure 5 Pie charts estimating the proportion of eyes receiving annual VF testing by (a) patient age (years), (b) glaucoma severity, and (c) glaucoma progression rate.

disease severity as measured by MD at baseline.

However, inspection of the curves fitted to the histogram and the 10th percentile values suggest fewer patients with early VF loss (green) are likely to have rapid progression. At the 10th percentile eyes with mild, moderate, and severe damage progress at rates of $-1.1,-1.5$, and $-1.3 \mathrm{~dB} /$ year, respectively.

The percentage of eyes receiving annual VF testing as stratified by age, severity of glaucoma, and rate of MD loss is shown in Figure 5. These data suggest that surveillance of patients with VF testing does not vary at all by age or stage of disease. More surprising, eyes losing vision quickly still mainly only receive annual VF testing.

\section{Discussion}

Rate of VF loss (dB/year) is a straightforward concept. For example, an eye diagnosed with an $\mathrm{MD}$ of $-8 \mathrm{~dB}$ will take 12 years to reach a level of assumed significant $\mathrm{VF}$ impairment (MD of $-20 \mathrm{~dB}$ ) if they progress at a rate of $-1 \mathrm{~dB} /$ year. This is an oversimplification because impactful localised central and binocular VF loss is sometimes not best measured by a single perimetric index like MD. Still, these calculations are clinically useful when managing a patient over time, especially when decisions 
need to be made about intensifying treatment. Recently, Chauhan and colleagues ${ }^{4}$ reported median (interquartile range) MD rate to be $-0.05(0.13,-0.30) \mathrm{dB} /$ year in 2324 unselected manifest and suspect glaucoma cases. Our study had an almost identical methodology and returned almost identical values albeit in a multicentre sample that was approximately sixfold larger. Confirmation that patients under routine glaucoma care demonstrate slow rates of VF progression is important to report because other studies have not been equivocal about this. ${ }^{4}$

Our wide-ranging longitudinal data provide new knowledge about how MD rate might be changing over time. Interestingly, the median rate of MD loss was faster in patients diagnosed in the first half of the decade as compared with the second. We speculate that this might be attributed to significant changes in the topical treatment of glaucoma patients and suspects which took place in the first part of that decade. The first randomised placebo-controlled trial to show effectiveness of prostaglandin treatment to preserve the VF has only recently been published, despite widespread use for many years. ${ }^{19}$ Owen and colleagues, ${ }^{20}$ using extensive GP prescription data, indicated prostaglandins became the dominant first-line glaucoma therapy in the UK in 2003, which conveniently coincides with the time point used to split our longitudinal data. On the other hand, in the absence of other information, the improvement in average MD rates might be attributed to changes in clinical management of cataract or other clinical management guideline changes during the 13-year period. Moreover, as is often the case, average values do not describe the entire distribution of the data. For example, the number of patients who were progressing rapidly did not change (Figure 3) and the 10th percentile of this distribution remained fixed throughout the period of follow-up (Figure 2). It would be fair to conclude that for the progression rates that matter most, there was no change over the time period we observed (Figure 3). This historical trend reflects there not being a dramatic change in treatment options for glaucoma, as there has been for other chronic eye diseases. ${ }^{21,22}$

Stratification of the sample by age revealed a clear relationship between the rate of MD loss worsening with older age. Patients over 70 years had considerably worse median rate of loss compared with younger patients. In this case, this large effect was applicable to the entire distribution of data (Figure 4) and this is noteworthy. This finding supports the results from other studies that have reported the same effect ${ }^{23-25}$ and refutes the finding in at least one other study (albeit in patients with untreated normal tension glaucoma only) that there is no association between age and rate of VF progression. ${ }^{26}$

The distribution of rate of MD loss also varied by baseline MD as indicated in the difference of the tails of the distribution rather than in the measures of central tendency in Figure 4. The 10th percentile for the distribution in the rates of loss from those patients with early VF loss indicated that rapid progressors are less likely to come from this group of patients when compared with those with moderate or advanced VF loss at diagnosis. This is revealing because it underlines the reduced risk of visual disability in those patients who are diagnosed in the earlier stages of the disease, not only because they have greater preserved vision to start with but they are, according to our data, less likely to progress rapidly than those patients who are diagnosed at a later stage. ${ }^{27-29}$ It is worth noting that the magnitude of this effect is, however, quite small. Moreover, precision of estimates of MD rates in individual eyes varies with VF damage and this has been established elsewhere. ${ }^{30}$ In other words, there is more variability in more damaged VFs, but this will likely not affect comparisons between such large groups of eyes.

A cross-sectional audit study conducted in several centres in England indicated that the large majority of patients have one VF examination per year ${ }^{31}$ and this seems true for the patients observed over the period of time in this study. Recent research evidence, reflected in clinical guidelines, has suggested that more frequent VF testing would help to identify rapidly progressing patients and this would have both clinical and potential health economic benefits. ${ }^{32,33}$ At the same time, this would require a shift in resources, clinician opinion, and patient views about automated perimetry. ${ }^{34,35}$ This study clearly shows a worse rate of VF loss was associated with older age and, albeit to a lesser extent, level of VF damage at diagnosis. Yet most patients simply receive the same diet of testing over time (Figure 5). In other words, there was no evidence that patients were stratified to receive more or less frequent examinations given their age, progression rate, or severity of glaucoma. In fact, it looks like there is a trend for patients with more rapid progression to less likely receive more frequent VF testing. There are certainly some interesting research studies that have recommended alternative types of VF follow-up schemes, in particular studies that have examined the clinical and cost-effectiveness of increased monitoring to detect fast progressors, ${ }^{32,36-38}$ but these have yet to translate to clinical practice. It would be interesting to consider stratification of VF resources to patients who might benefit more or less from them and this ought to be a question for future prospective studies.

The main problem with the design of this study is the absence of any clinical indicators on the eyes other than the VF results. So, for example, we had no information about exact diagnosis, intraocular pressure, optic nerve head characteristics, individual patient history, or other risk factors. Likewise, we had no information about 
types of treatment and concomitant eye disease. At the same time, these data represent unselected people in glaucoma clinics who are receiving routine care. Moreover, the sheer size of the data provides interesting insights that might not be uncovered by controlled prospective studies on smaller numbers of people. This study has other notable limitations: it was multicentre but the sampling was not carried out systematically and the data are not particularly current because the extractions were carried out in 2012. Furthermore, HFA reliability indices are used to exclude poorly carried out examinations in clinical practice, but these were not used in this study because many were missing from the original database.

In conclusion, the results from this study suggest that patients in clinics in England, on average, experience a relatively slow rate of VF deterioration. It is important to recognise, however, that a proportion of eyes progress at a rate sufficiently fast that is likely to result in a visual impairment classification within their lifetimes.

Furthermore, although this study found that median rates of MD loss appear to be declining, it is of note that this trend was not evident in patients who matter the most; that is, individuals with medium to fast rates of VF loss. Of course, our results refer to a patient population and have little bearing on the management of individuals. Yet, this study illustrates the use of large VF databases to monitor and audit service delivery of glaucoma treatment. Digital records from automated perimetry are amenable to electronic audits and this report seeks to motivate initiatives for glaucoma similar to those put into place for other eye diseases. These data can then be used in combination with health economic techniques in order to investigate potential efficiency gains within the treatment of glaucoma and potentially reduce the burden of visual impairment in the UK.

\section{Summary}

\section{What was known before}

- There is a lack of information regarding rates of glaucomatous visual field progression and how they may be changing over time. Such information is vital in understanding the efficiency of service delivery for glaucoma in the UK.

\section{What this study adds}

- Average visual field progression rates have only improved marginally over the last decade. The proportion of fast progressors has not reduced. Visual fields of older eyes are, on average, found to progress faster than younger eyes. There is no evidence of patients being stratified to receive more or less frequent visual field examination, given their age, severity of glaucoma, or progression speed.

\section{Conflict of interest}

The authors declare no conflict of interest.

\section{Acknowledgements}

We would like to thank Andrew McNaught (Department of Ophthalmology, Gloucestershire Hospitals NHS Foundation Trust, Cheltenham and Cranfield University, Bedford), James Kirwan (Department of Ophthalmology, Queen Alexandra Hospital, Portsmouth), and Nitin Anand (Calderdale and Huddersfield NHS Foundation Trust) for helping to provide access to visual field data from their respective hospitals. We also thank Richard Russell for his efforts with the initial data analysis.

\section{References}

1 Heijl A, Buchholz P, Norrgren G, Bengtsson B. Rates of visual field progression in clinical glaucoma care. Acta Ophthalmol 2012; 91(5): 406-412.

2 Chauhan BC. Group CGS Canadian Glaucoma Study: 1. Study design, baseline characteristics, and preliminary analyses. Can J Ophthalmol 2006; 41(5): 566-575.

3 De Moraes CGV, Juthani VJ, Liebmann JM, Teng CC, Tello C, Susanna R et al. Risk factors for visual field progression in treated glaucoma. Arch Ophthalmol 2011; 129(5): 562-568.

4 Chauhan BC, Malik R, Shuba LM, Rafuse PE, Nicolela MT, Artes PH. Rates of glaucomatous visual field change in a large clinical population. Invest Ophthalmol Vis Sci 2014; 55(7): 4135-4143.

5 Keenan T, Johnston R, Donachie P, Sparrow J, Stratton I, Scanlon P. United Kingdom National Ophthalmology Database Study: Diabetic Retinopathy; Report 1: prevalence of centre-involving diabetic macular oedema and other grades of maculopathy and retinopathy in hospital eye services. Eye 2013; 27(12): 1397-1404.

6 Tufail A, Xing W, Johnston R, Akerele T, McKibbin M, Downey $\mathrm{L}$ et al. The neovascular age-related macular degeneration database: multicenter study of 92976 ranibizumab injections. Ophthalmology 2014; 121(5): 1092-1101.

7 Sparrow J, Taylor H, Qureshi K, Smith R, Birnie K, Johnston R. The Cataract National Dataset electronic multi-centre audit of 55567 operations: risk indicators for monocular visual acuity outcomes. Eye 2012; 26(6): 821-826.

8 Butt T, Lee A, Lee C, Tufail A, Xing W, Johnston RL et al. The cost-effectiveness of initiating ranibizumab therapy in eyes with neovascular AMD with good vision: an economic model using real-world outcomes. BMJ Open 2015; 5(5): e006535.

9 Boodhna T, Crabb DP. Disease severity in newly diagnosed glaucoma patients with visual field loss: trends from more than a decade of data. Ophthalmic Physiol Opt 2014; 35(2): 225-230.

10 Saunders LJ, Russell RA, Kirwan JF, McNaught AI, Crabb DP. Examining visual field loss in patients in glaucoma clinics during their predicted remaining lifetime. Invest Ophthalmol Vis Sci 2014; 55(1): 102-109. 
11 Bateman D, Clark R, Azuara-Blanco A, Bain M, Forrest J. The effects of new topical treatments on management of glaucoma in Scotland: an examination of ophthalmological health care. Br J Ophthalmol 2002; 86(5): 551-554.

12 Saunders LJ, Russell RA, Crabb DP. Practical landmarks for visual field disability in glaucoma. Br J Ophthalmol 2012; 96: 1185-1189.

13 Wild JM, Dengler-Harles M, Searle AET, O'Neill EC, Crews SJ. The influence of the learning effect on automated perimetry in patients with suspected glaucoma. Acta Ophthalmol (Copenh) 1989; 67: 537-545.

14 Heijl A, Lindgren G, Olsson J. The effect of perimetric experience in normal subjects. Arch Ophthalmol 1989; 107(1): 81-86.

15 Heijl A, Bengtsson B. The effect of perimetric experience in patients with glaucoma. Arch Ophthalmol 1996; 114(1): 19-22.

16 Jansonius N. On the accuracy of measuring rates of visual field change in glaucoma. Br J Ophthalmol 2010; 94(10): 1404-1405.

17 Hodapp E, Parrish RI, Anderson DR. Clinical Decisions in Glaucoma. The CV Mosby: St Louis, MO, 1993.

18 R Development Core Team. $R$ : A language and environment for statistical computing. R Foundation for Statistical Computing: Vienna, Austria, 2008.

19 Garway-Heath DF, Crabb DP, Bunce C, Lascaratos G, Amalfitano F, Anand $\mathrm{N}$ et al. Latanoprost for open-angle glaucoma (UKGTS): a randomised, multicentre, placebocontrolled trial. Lancet 2014; 385(9975): 1295-1304.

20 Owen CG, Carey I, De Wilde S, Whincup P, Wormald R, Cook D. The epidemiology of medical treatment for glaucoma and ocular hypertension in the United Kingdom: 1994 to 2003. Br J Ophthalmol 2006; 90(7): 861-868.

21 Simó R, Hernández C. Intravitreous anti-VEGF for diabetic retinopathy: hopes and fears for a new therapeutic strategy. Diabetologia 2008; 51(9): 1574-1580.

22 Holekamp NM, Liu Y, Yeh W-S, Chia Y, Kiss S, Almony A et al. Clinical utilization of anti-VEGF agents and disease monitoring in neovascular age-related macular degeneration. Am J Ophthalmol 2014; 157(4): 825-833 e1.

23 Chauhan BC, Mikelberg FS, Balaszi AG, LeBlanc RP, Lesk MR, Trope GE. Canadian Glaucoma Study: 2. risk factors for the progression of open-angle glaucoma. Arch Ophthalmol 2008; 126(8): 1030-1036.

24 Leske MC, Heijl A, Hyman L, Bengtsson B, Dong L, Yang Z. Predictors of long-term progression in the early manifest glaucoma trial. Ophthalmology 2007; 114(11): 1965-1972.

25 Nouri-Mahdavi K, Hoffman D, Coleman AL, Liu G, Li G, Gaasterland D et al. Predictive factors for glaucomatous visual field progression in the Advanced Glaucoma Intervention Study. Ophthalmology 2004; 111(9): 1627-1635.

26 Drance S, Anderson DR, Schulzer M. Group CN-TGS Risk factors for progression of visual field abnormalities in normal-tension glaucoma. Am J Ophthalmol 2001; 131(6) 699-708.

27 Prata TS, De Moraes CG, Teng CC, Tello C, Ritch R, Liebmann JM. Factors affecting rates of visual field progression in glaucoma patients with optic disc hemorrhage. Ophthalmology 2010; 117(1): 24-29.

28 Leske MC, Heijl A, Hussein M, Bengtsson B, Hyman L, Komaroff E. Factors for glaucoma progression and the effect of treatment: the early manifest glaucoma trial. Arch Ophthalmol 2003; 121(1): 48-56.

29 Wesselink C, Stoutenbeek R, Jansonius N. Incorporating life expectancy in glaucoma care. Eye 2011; 25(12): 1575-1580.

30 Russell RA, Crabb DP, Malik R, Garway-Heath DF. The relationship between variability and sensitivity in large-scale longitudinal visual field data. Invest Ophthalmol Vis Sci 2012; 53(10): 5985-5990.

31 Fung SS, Lemer C, Russell RA, Malik R, Crabb DP. Are practical recommendations practiced? A national multicentre cross-sectional study on frequency of visual field testing in glaucoma. Br J Ophthalmol 2013; 97(7): 843-847.

32 Crabb D, Russell R, Malik R, Anand N, Baker H, Boodhna T et al. Frequency of visual field testing when monitoring patients newly diagnosed with glaucoma: mixed methods and modelling. Health Serv Deliv Res 2014; 2(27): 49-77.

33 European Glaucoma Society. Terminology and Guidelines for Glaucoma. 3rd edn. Editrice Dogma: Savona, Italy, 2008.

34 Malik R, Baker H, Russell RA, Crabb DP. A survey of attitudes of glaucoma subspecialists in England and Wales to visual field test intervals in relation to NICE guidelines. BMJ Open 2013; 3(5): e002067.

35 Glen FC, Baker H, Crabb DP. A qualitative investigation into patients' views on visual field testing for glaucoma monitoring. BMJ Open 2014; 4(1): e003996.

36 Chauhan BC, Garway-Heath DF, Goni FJ, Rossetti L, Bengtsson B, Viswanathan AC et al. Practical recommendations for measuring rates of visual field change in glaucoma. Br J Ophthalmol 2008; 92(4): 569-573.

37 Crabb DP, Garway-Heath DF. Intervals between visual field tests when monitoring the glaucomatous patient: wait-andsee approach. Invest Ophthalmol Vis Sci 2012; 53(6): 2770-2776.

38 Jansonius NM. Progression detection in glaucoma can be made more efficient by using a variable interval between successive visual field tests. Graefes Arch Clin Exp Ophthalmol 2007; 245(11): 1647-1651. 